\title{
Optimized imaging of the suprachoroidal space with swept-source OCT
}

\author{
Joel Hanhart, Yaakov Rozenman \\ Department of Ophthalmology, Shaare Zedek Medical Center, Jerusalem, \\ IsraelAbstract
}

\begin{abstract}
Purpose: To compare enhanced depth imaging (EDI) and non-EDI swept source optical coherence tomography (SS-OCT) in their ability to capture the suprachoroidal space (SCS).

Materials and methods: Twenty volunteers with a minimum age of 18 years without any ocular pathology and refractive error below \pm 2 diopters underwent SS-OCT foveal scanning, with and without EDI. Masked averaged B-scan lines were analyzed for presence of the SCS. When the SCS was seen, the percentage of the scan on which this structure could be unequivocally observed was measured. Scores obtained from the images taken with or without EDI were then compared.

Results: Thirty-seven eyes were analysed, since three eyes of three different patients were eliminated, as the outer border of the choroid was insufficiently delineated with both modalities. The SCS was not detected at all on 14 pictures (37.8\%) obtained by non-EDI SS-OCT and 9 pictures (24.3\%) obtained by EDI SS-OCT. When the SCS was detected with both modalities, it was observable on $27.2 \pm 24.2 \%$ of the scan without EDI and $40.4 \pm 30.3$ of the scan with EDI $(p<.001)$

Conclusions: EDI SS-OCT enables a more frequent and extensive visualization of the suprachoroidal space than non-EDI SS-OCT. This new approach could be considered as the most accurate modality to currently visualize the SCS in vivo.
\end{abstract}

Keywords: choroid, EDI, suprachoroidal layer, swept-source OCT

\section{Introduction}

The suprachoroidal space (SCS) is receiving increasing attention as its potential role in the diagnosis and treatment of various retinal conditions is being recognized.'

Enhanced depth imaging (EDI), a modification of the regular acquisition technique, was originally described for spectral-domain optical coherence tomography (SD-OCT) in order to improve visualization of deeper structures within the choroid. ${ }^{2}$ EDI improved visualization of the choroidoscleral interface.

Correspondence: Joel Hanhart, MD, Department of Ophthalmology, Shaare Zedek Medical Center, 12 Beyt Street, Jerusalem 91031, Israel.

E-mail: hanhart@szmc.org.il 
There are, however, limitations in the ability of EDI SD-OCT to capture details of the outer choroidal border. ${ }^{3,4}$ On the other hand, the SCS has been captured in health and disease by using swept-source OCT (SS-OCT). ${ }^{5}$

In this research, we aim to compare non-EDI and EDI-OCT in their ability to capture the SCS in healthy subjects and thereby attempt to improve the visualization of such an important structure. For this, we propose to evaluate the proportion of eyes in which the SCS is identified, applying the EDI acquisition protocol. When the SCS is seen, we aim to quantify on what percentage of the macular scan it is patent and draw a comparison between both imaging approaches.

\section{Materials and methods}

Ethics approval for this prospective, interventional case series was obtained from the Ethics Committee at Shaare Zedek Medical Center.

\section{Participants and imaging protocol}

Healthy volunteers with a minimum age of 18 years and refractive error below $\pm 2 \mathrm{D}$, without any ocular pathology, were prospectively recruited at the Department of Ophthalmology, Shaare Zedek Medical Center, Jerusalem. Without pupil dilatation, they were imaged with DRI OCT-1 Atlantis (Topcon Medical Systems Inc., Oakland, NJ; software version 9.10; 1,050 nm central wavelength) in random order by a single, experienced operator. For each eye, averaged B-scan lines at the fovea were obtained by two techniques, with the hyperreflectivity at the bottom of the foveal pit as an indicator of correct scan position. Non-EDI pictures were first taken according to the instructions of the manufacturer. Then, EDI scans were obtained, the device being pushed close enough to the eye to create an inverted image near the top of the display. Enough separation from the top of the display was used to avoid image ambiguity from image folding with respect to zero depth.

\section{Image evaluation}

All pictures were transferred to the image software. Those obtained by EDI, presented originally with the choroid up and the retina down, were inverted, so masking of the acquisition technique could be guaranteed. For each image, a retina specialist noticed if a hyporeflective band corresponding to the described location of the SCS was identified. When the SCS was seen, the percentage of the scan (from Bruch's membrane opening at the temporal border of the optic nerve head to the temporal edge of the scan) on which this structure could be unequivocally observed was measured. Scores obtained from the images taken with or without EDI were then compared. 


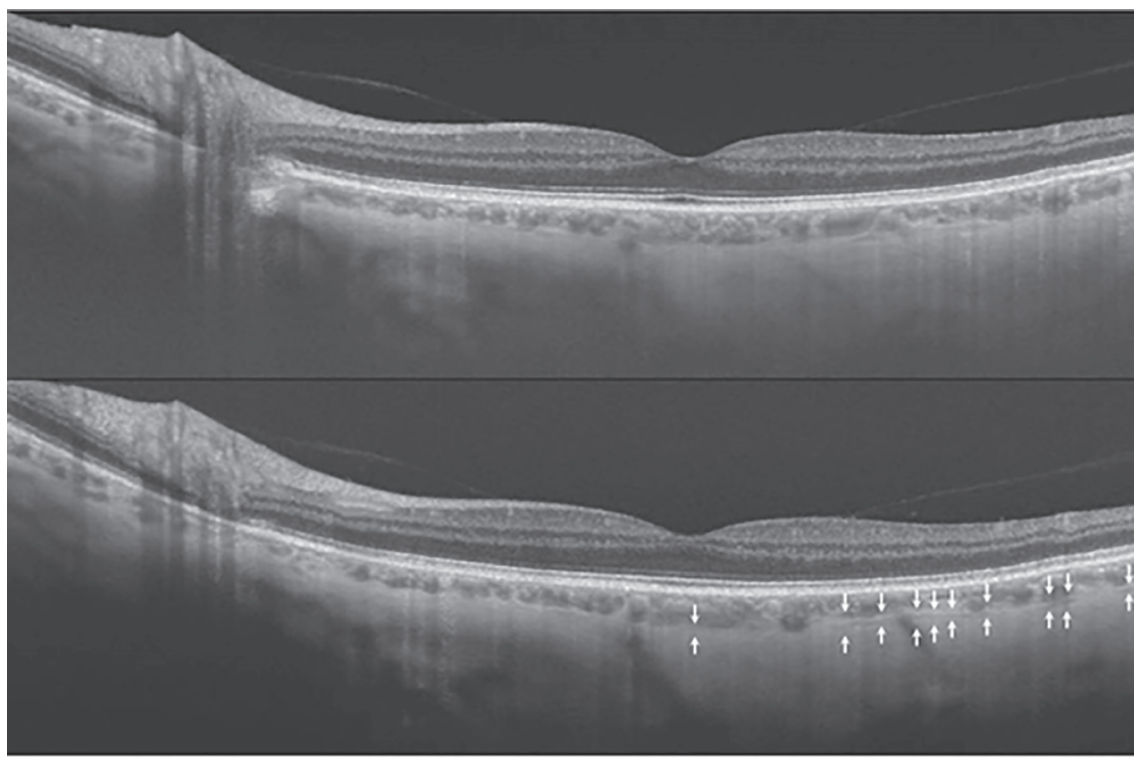

Fig. 1. EDI-OCT enabling visualization of the SCS. Using SS-OCT without EDI, the SCS was not detected. EDI allows following the SCS on the temporal macula.

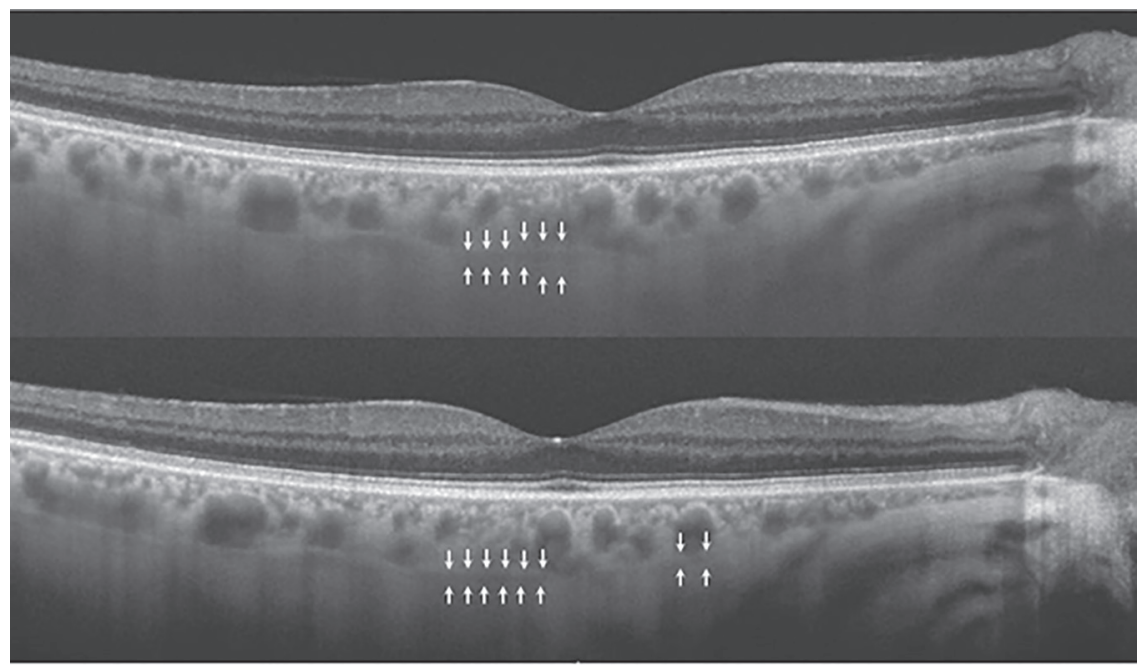

Fig. 2. Mild improvement in visualization of the SCS with EDI. While the SCS was captured with non-EDI SS-OCT, applying EDI enabled to detect it to a greater extent. 


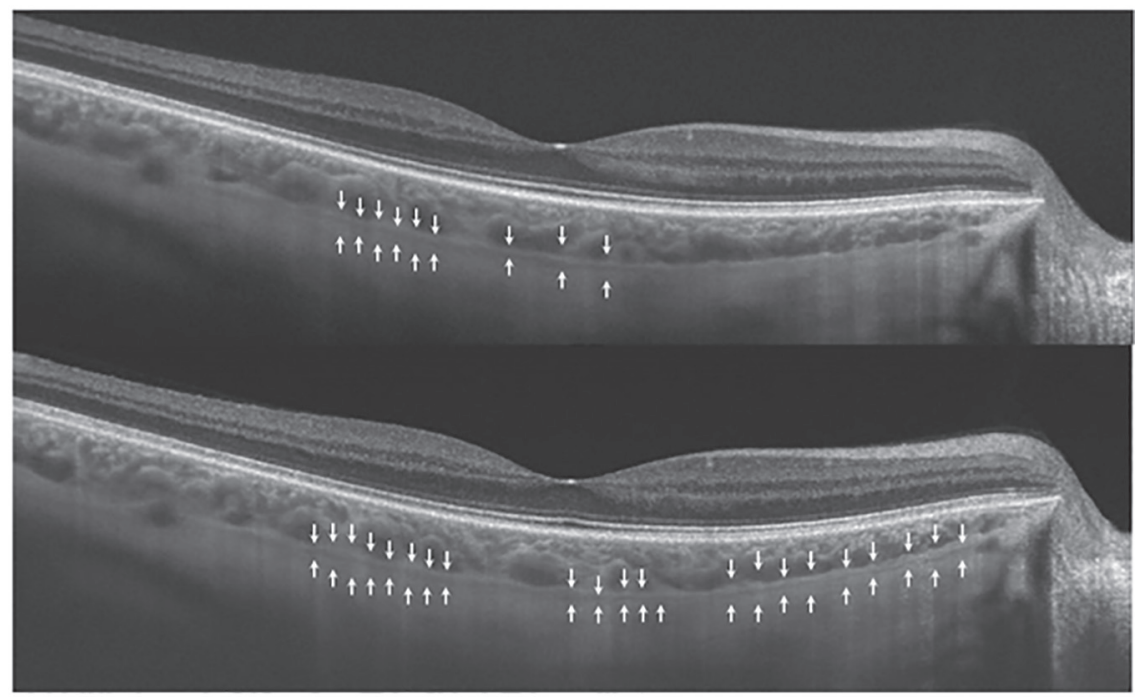

Fig. 3. Considerable improvement in visualization of the SCS with EDI. The SCS could be followed over some part of the scan length without EDI. However, by applying the EDI technique, it was visualized until the opening of Bruch's membrane.

\section{Results}

Forty eyes of 20 volunteers were included. The mean age ( \pm standard deviation) was $37.7 \pm 8.5$ years. EDI images were easily obtained for all eyes. There was no significant difference in the number of averaged eyes between the non-EDI (93.4 $\pm 6.5)$ and EDI series $(91.9 \pm 9.6 ; p=0.4)$. Three eyes of three different patients were eliminated, as the outer border of the choroid was insufficiently delineated with both modalities. SCS was not detected in 14 pictures (37.8\%) obtained by non-EDI SS-OCT and 9 pictures (24.3\%) obtained by EDI SS-OCT. In the five eyes in which SCS was captured by EDI SS-OCT only, SCS could be followed over $10 \pm$ $1.6 \%$ of the scan (range: $5 \%-15 \%$ ).

When SCS was detected with both modalities, it was observable on $27.2 \pm$ $24.2 \%$ of the scan without EDI (range: $5 \%-70 \%$ ) and $40.4 \pm 30.3 \%$ of the scan with EDI (range: $5 \%-90 \%)$. The difference in proportion of observable SCS was found to be statistically significant $(p<0.001)$.

\section{Discussion}

Applied to SS-OCT, EDI increases the proportion of eyes in which the SCS is detectable (from $62.2 \%$ to $75.7 \%$ ) (Fig. 1).

EDI also enables to see larger parts of the SCS than non-EDI SS-OCT (13.2\% more of the total scan length) (Figs. 2 and 3). 
In the young population we studied, SS-OCT obtained better results than previously reported studies with EDI SD-OCT.6-8 In our series, the SCS was identified in $62.2 \%$ of the eyes, EDI increasing the detection rate to $75.7 \%$.

Michalewska et al. assessing the suprachoroidal layer and space using non-EDI SS-OCT were able to visualize the SCS in $20 \%$ of eyes with neovascular age-related macular degeneration (AMD), $50 \%$ of eyes with dry AMD, and $50 \%$ of those with full-thickness macular holes. ${ }^{5}$ Remarkably, they identified the SCS in only one healthy and one highly myopic eye (5\% of each group), a rate lower than EDI SD-OCT. ${ }^{5-8}$

With EDI SS-OCT, we were able to observe the SCS more frequently than what was reported by researchers using EDI SD-OCT or non-EDI SS-OCT.

It is likely that in the near future, the SCS will become a routine component in the diagnosis, monitoring, and treatment of retinal diseases, and it is likely that new discoveries regarding its potential uses will be made in the near future. ${ }^{1}$

Our results, based on a small number of patients, should be validated by larger studies, with other SS-OCT devices and involvement of various observers, in order to analyze the SCS not only in health but also in disease.

In conclusion, this study indicates that the EDI technique applied to SS-OCT enhances visualization of the SCS. Currently, this approach is probably the most accurate to detect and study the SCS.

\section{Conflict of interest}

The authors report no conflict of interest.

\section{References}

1. Moisseiev E, Loewenstein A, Yiu G. The suprachoroidal space: from potential space to a space with potential. Clin Ophthalmol. 2016;10:173-178.

2. Spaide RF, Koizumi H, Pozzoni MC. Enhanced depth imaging spectral-domain optical coherence tomography. Am J Ophthalmol. 2008;146(4):496-500.

3. Copete S, Flores-Moreno I, Montero JA, Duker JS, Ruiz-Moreno JM. Direct comparison of spectraldomain and swept-source OCT in the measurement of choroidal thickness in normal eyes. $\mathrm{Br}$ J Ophthalmol. 2014;98(3):334-338.

4. Adhi M, Liu JJ, Qavi AH, Grulkowski I, Fujimoto JG, Duker JS. Enhanced visualization of the choroido-scleral interface using swept-source OCT. Ophthalmic Surg Lasers Imaging Retina. 2013;44(6 Suppl):S40-S42.

5. Michalewska Z, Michalewski J, Nawrocka Z, Dulczewska-Cichecka K, Nawrocki J. Suprachoroidal layer and suprachoroidal space delineating the outer margin of the choroid in swept-source optical coherence tomography. Retina. 2015;35(2):244-249.

6. Yiu G, Pecen P, Sarin N, et al. Characterization of the choroid-scleral junction and suprachoroidal layer in healthy individuals on enhanced-depth imaging optical coherence tomography. JAMA Ophthalmol. 2014;132(2):174-181.

7. Kim JH, Chang YS, Kim JW, Lee TG, Lew YJ. Imaging suprachoroidal layer in exudative age-related macular degeneration. Curr Eye Res. 2015;41(5):715-720. 
8. Boonarpha N, Zheng Y, Stangos AN, et al. Standardization of choroidal thickness measurements using enhanced depth imaging optical coherence tomography. Int J Ophthalmol. 2015;8(3):484-491. 